Thorax, 1977, 32, 505-508

\title{
Primary pulmonary sporotrichosis with unusual fungal morphology
}

\author{
S. D. BERSON AND F. A. BRANDT
}

From the Departments of Pathology, National Research Institute for Occupational Diseases of the South African Medical Research Council, Johannesburg and the South African Institute for Medical Research, Johannesburg, South Africa

Berson, S. D., and Brandt, F. A. (1977). Thorax, 32, 505-508. Primary pulmonary sporotrichosis with unusual fungal morphology. Primary pulmonary sporotrichosis is a rare disease. Comstock and Wolson (1975) were able to trace approximately 30 such cases. We report a further case; to our knowledge, the various stages in the development of sporotrichosis, from the cigar body to the mature asteroid body with radiations which were present in our case, have not been so well demonstrated in a single case.

In 1898 Schenck published an article entitled 'On refractory subcutaneous abscesses caused by a fungus possibly related to the sporotrichia'. In 1947 the Transvaal Chamber of Mines reported an epidemic of sporotrichosis involving nearly 3000 South African gold miners, of whom only five did not have the classic cutaneous and lymphangitic form, thus emphasising Schenck's (1898) original observations.

Various clinical types of infection are recognised as being due to this world-wide distributed, saprophytic biphasic organism which is found among plants, insects, animals, and man, and may be transmitted by the bites of insects, birds, and both wild and domestic animals. The primary pulmonary form of sporotrichosis is the rarest clinical type. The spores of Sporotrichium schenckii are 2-3 $\mu$ in diameter and are therefore small enough to penetrate to the alveoli, and yet Mohr et al. (1972) were able to report fewer than 30 documented cases of primary pulmonary infection, an observation confirmed by Comstock and Wolson's (1975) report. This present case report describes the unexpected finding of primary pulmonary sporotrichosis with most unusual fungal morphological changes.

\section{Case report}

In November 1972, a Malawian gold miner aged 36 years was admitted to hospital complaining of general debility and possibly a mumps infection. No definite diagnosis was made at this time. In January 1973 he sustained a contusion to his right knee joint but no record exists as to where the injury occurred, on surface or underground, and in what circumstances. The wound, however, healed rapidly, leaving no scar.

He developed a doubtful parotitis in May 1973 and during the next three months he was admitted to hospital at monthly intervals complaining of general debility but again no definitive diagnosis was made. Chest radiographs in July 1973 showed a diffuse increase in lung markings. However, in August 1973 a normal chest radiograph was reported. Later in August he was admitted to hospital with a bleeding tongue and died six days later in uraemia.

Relevant macroscopic and microscopic postmortem findings included the following:

Calcified and caseous root glands at the hila of the lungs with features consistent with an active tuberculous lymphadenitis although acid and alcohol fast bacilli were not demonstrated in suitably stained preparations. In addition, acute abscess formation was present in which fungal elements consistent with $S$. schenckii were present.

The lungs showed foci of bronchopneumonic consolidation with the histological features of an acute suppurative bronchopneumonia with organisation in part. Once again no acid and alcohol fast organisms were identified. However, fungal elements were present consistent with $S$. schenckii. The kidneys were the seat of a chronic pyelonephritis.

Examination of the morphology of the fungal infection present showed a most unexpected spectrum of changes which included the classical 
asteroid body associated with $S$. schenckii. In addition, spores with multiple budding, spores with budding and filament formation, cigar bodies clumped within the asteroid itself, and typical filamentous clumping of $S$. schenckii were all noted. These features are demonstrated in Figs 1 to 5. Confirmation that the organism was $S$. schenckii was obtained by a positive specific fluorescent antibody test, carried out by Dr. Kaplan (Kaplan and Ivens, 1960; Kaplan and Kraft, 1969). No other systemic evidence of fungal infection or tuberculosis was noted. This case has therefore been considered to represent one of primary pulmonary sporotrichosis.

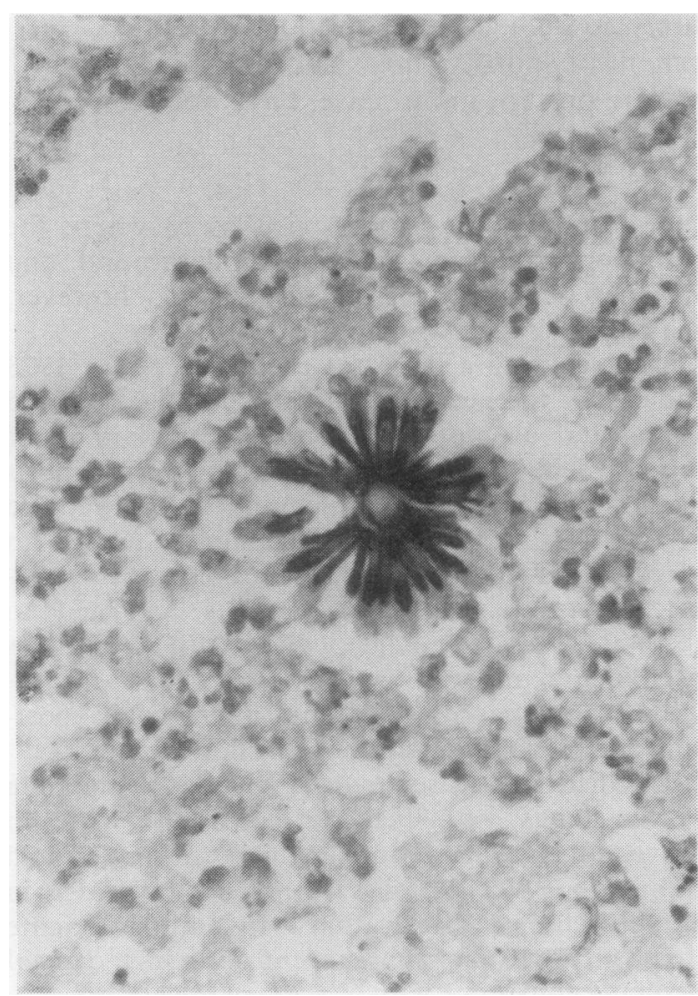

Fig. 1 Classical asteroid body with doubly refractile wall and radiating rosette formation.

\section{Discussion}

Comstock and Wolson (1975) discuss the radiological changes in sporotrichosis and state that the pulmonary findings depend upon whether or not the lung is the only site of infection. If there are no other areas of involvement, the pulmonary findings may be indistinguishable from those of

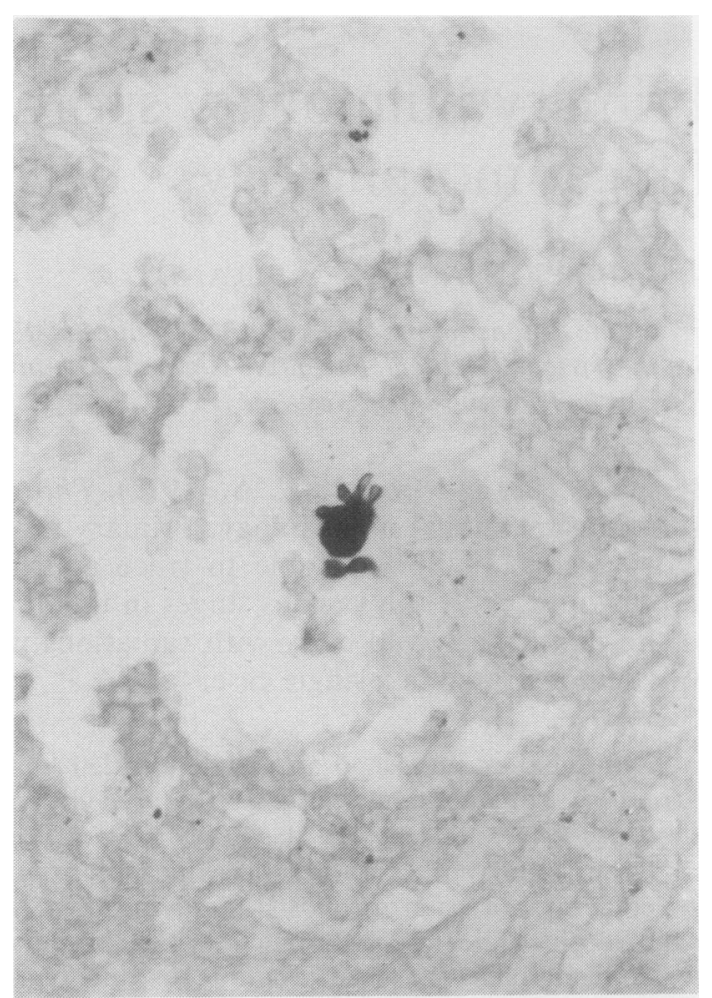

Fig. 2 Asteroid body in which the spore demonstrates multiple budding. The apparent absence of radiating rosette formation is due to the photographic filter used to accentuate the budding process.

secondary tuberculosis. Our case did show histological features consistent with a tuberculous infection but unexpectedly also showed pulmonary sporotrichosis. The possible source of infection was not determined but no skin involvement was present.

The interest of our case is thus not in the clinical and radiographic patterns that pulmonary sporotrichosis may demonstrate but rather in the complete spectrum of the developmental cycle $N$ present; to our knowledge, the various stages from the mature asteroid body with radiation to the cigar body formation have not been demonstrated in one clinical case.

In the normal developmental cycle this fungus is considered to pass through the following phases in vivo, as demonstrated by animal experiments (Brandt and Van Niekerk, 1968 and 1969):

1 The small elliptical spore is introduced into the body.

2 Within 24 hours there is a histiocytic tissue response with phagocytosis of some spores, 


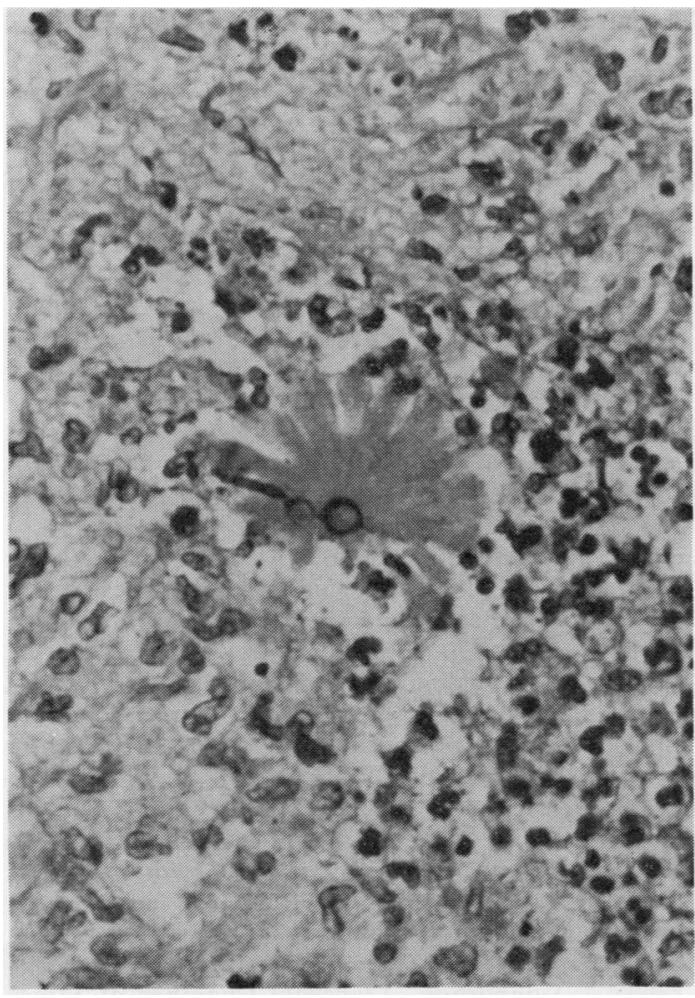

Fig. 3 Asteroid showing budding and filament formation.

and superficially at this stage there is a resemblance to histoplasmosis histologically.

3 (a) The ingested spores apparently are rendered non-viable and play no further rôle in the pathogenesis of the disease.

(b) The non-ingested spores tend to bud with a simultaneous local acute inflammatory cell reaction consisting of polymorphonuclear leucocytes and lymphocytes only at this stage.

4 The bud elongates, forming classical cigar bodies with an associated diffuse acute inflammatory reaction too extensive to be designated a microabscess.

5 In some cases the cigar body rounds off and forms a double contour to produce the classic large hyaline spore with two forms of tissue reaction-

(a) the formation of microabscess; and

(b) classical radiating material which is considered to be tissue and not fungal in origin.

6 Remnants of other cigar bodies in various

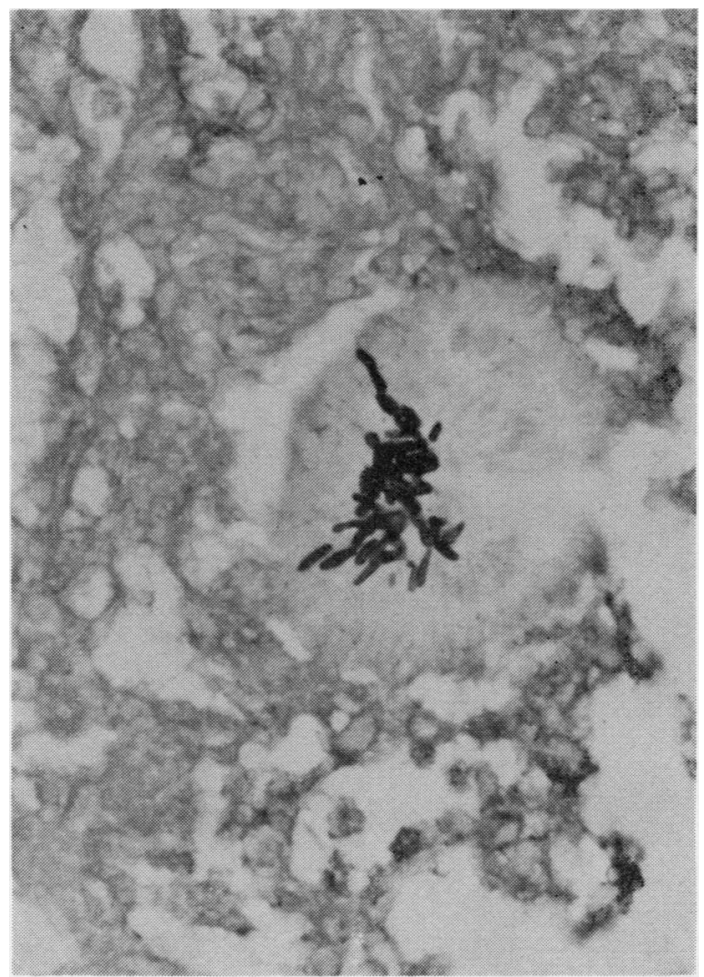

Fig. 4 Asteroid showing the most unusual feature of cigar body clumping within the asteroid itself.

stages of disintegration may be observed with no striking tissue response.

The above changes occur over a two to three week interval, and thus the changes noted in our case must have occurred in vivo and not in the 72 hours which elapsed between death and the postmortem examination.

One of us (FAB) had the opportunity of examining a clinical case of disseminated sporotrichosis in which the most advanced developmental stage was the cigar body, with no evidence of asteroid formation.

Experience shows that in chronic skin involvement, the asteroid body will be found in the majority of cases if searched for diligently enough.

Our present case shows a complete spectrum in the developmental cycle in one organ only and can justifiably be classified as one of primary pulmonary sporotrichosis.

It should be noted that different organ involvement may explain different tissue responses, and this concept certainly warrants further investiga- 


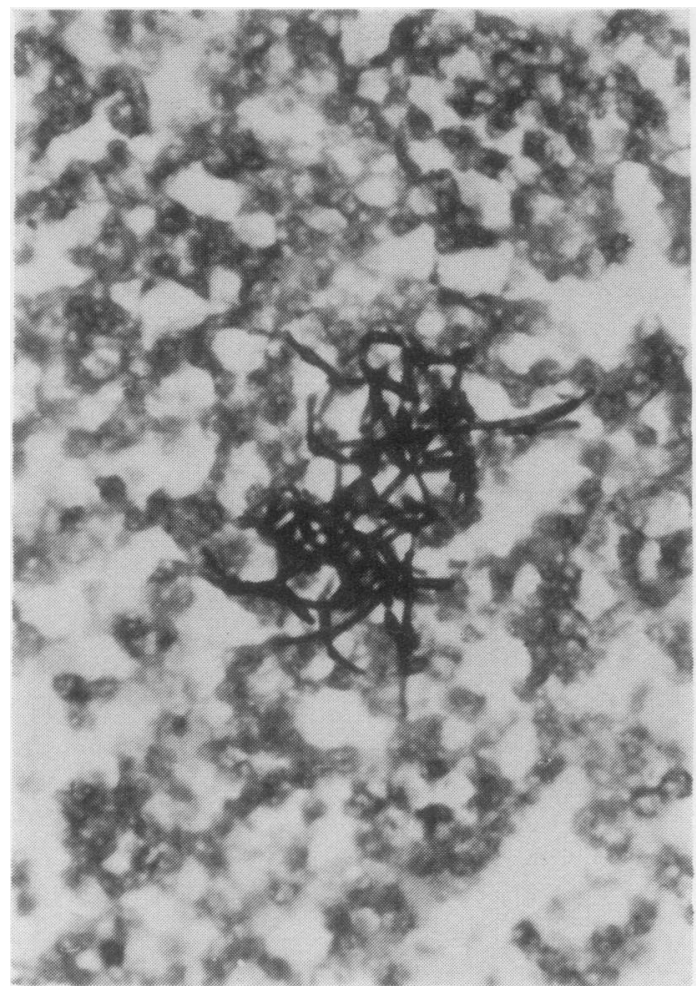

Fig. 5 Typical filamentous clumping of S. schenckii.

tion. No definite opinion can be given on the rôle the pulmonary infection played in the ultimate cause of death, which was considered to be due to chronic pyelonephrosis.

\section{References}

Brandt, F. A., and Van Niekerk, V. (1968). An atypical strain of sporothrix from South Africa. Journal of Pathology and Bacteriology, 96, 39-44.

Brandt, F. A., and Van Niekerk, V. (1969). A case of disseminating sporotrichosis from South Africa. Sabouraudia; Journal of the International Society for Human and Animal Mycology, 7, 46-50.

Comstock, C., and Wolson, A. H. (1975). Roentgenology of sporotrichosis. American Journal of Roentgenology, Radium Therapy and Nuclear Medicine, 125, 651-655.

Kaplan, W., and Ivens, M. S. (1960). Fluorescent antibody staining of Sporotrichium schenckii in cultures and clinical materials. Journal of Investigative Dermatology, 35, 151-159.

Kaplan, W., and Kraft, D. E. (1969). Demonstration of pathogenic fungi in formalin-fixed tissues by immunofluorescence. American Journal of Clinical Pathology, 52, 420-432.

Mohr, J. A., Patterson, C. D., Eaton, B. G., Rhoades, E. R., and Nichols N. B. (1972). Primary pulmonary sporotrichosis. American Review of Respiratory Disease, 106, 260-264.

Schenck, B. R. (1898). On refractory subcutaneous abscesses caused by a fungus possibly related to the sporotrichia. Bulletin of the Johns Hopkins Hospital, 9, 286-290.

Transvaal Mine Medical Officers' Association (1947). Sporotrichosis Infection on Mines of the Witwatersrand: A Symposium. Transvaal Chamber of Mines Johannesburg.

Requests for reprints to: The Librarian, National Research Institute for Occupational Diseases, PO Box 4788, Johannesburg 2000, South Africa. 